\title{
Multi-band structure of a coupling constant for quantum bound states of a generalized nonlinear Schrödinger model
}

\author{
B. Basu-Mallick ${ }^{\text {a }}$, Tanaya Bhattacharyya ${ }^{\mathrm{a}, *}$, Diptiman Sen ${ }^{\mathrm{b}}$ \\ a Theory Group, Saha Institute of Nuclear Physics, 1/AF Bidhan Nagar, Kolkata 700 064, India \\ ${ }^{\mathrm{b}}$ Centre for High Energy Physics, Indian Institute of Science, Bangalore 560012, India
}

\begin{abstract}
Using Bethe ansatz we study $N$-body bound states of a generalized nonlinear Schrödinger model with two coupling constants $c$ and $\eta$. We find that bound states exist for all values of $c$ but only within certain ranges (called bands) of $\eta$. These ranges are governed by Farey sequences in number theory.
\end{abstract}

Keywords: Nonlinear Schrödinger model; Coordinate Bethe ansatz; Bound states

\section{Introduction}

Bound states in integrable quantum field theory models in $1+1$ dimensions have been studied extensively for many years [1-12]. The quantum bound states are usually constructed by using either the coordinate Bethe ansatz or the algebraic Bethe ansatz. For an integrable nonrelativistic Hamiltonian, the coordi-

\footnotetext{
* Corresponding author.

E-mail addresses: biru@theory.saha.ernet.in

(B. Basu-Mallick), tanaya@theory.saha.ernet.in

(T. Bhattacharyya), diptiman @ cts.iisc.ernet.in (D. Sen).
}

nate Bethe ansatz can yield the exact eigenfunctions in the coordinate representation. If such an eigenfunction decays sufficiently fast when any of the particle coordinates tends towards infinity (keeping the center of mass coordinate fixed), we call such a localized square-integrable eigenfunction a quantum bound state. It is also possible to construct quantum bound states using the algebraic Bethe ansatz, by choosing appropriate distributions of the spectral parameter in the complex plane [3-5]. It is usually found that localized quantum bound states of various integrable models, including the well-known nonlinear Schrödinger model (NLS) and the sine-Gordon model, have positive binding energy [1-6]. 
In this Letter, we will study the quantum bound states of a generalized NLS model. Classical and quantum versions of this type of models have found applications in different areas of physics like circularly polarized nonlinear Alfvén waves in a plasma $[13,14]$ and quantum properties of solitons in optical fibers [15]. The Hamiltonian of the generalized NLS model in its second quantized form is given by [7]

$$
\begin{aligned}
H= & \int_{-\infty}^{+\infty} d x\left[\hbar \psi_{x}^{\dagger} \psi_{x}+c \psi^{\dagger 2} \psi^{2}\right. \\
& \left.+i \eta\left\{\psi^{\dagger 2} \psi \psi_{x}-\psi_{x}^{\dagger} \psi^{\dagger} \psi^{2}\right\}\right],
\end{aligned}
$$

where the subscripts $t$ and $x$ denote partial derivatives with respect to time and space respectively, $\eta$ and $c$ are real coupling constants, and we have set the particle mass $m=1 / 2$. The coupling constant $\eta$ is dimensionless whereas $c$ has the dimension of inverse length. The field operators $\psi(x, t), \psi^{\dagger}(x, t)$ obey the equal time commutation relations, $[\psi(x, t), \psi(y, t)]=$ $\left[\psi^{\dagger}(x, t), \psi^{\dagger}(y, t)\right]=0$, and $\left[\psi(x, t), \psi^{\dagger}(y, t)\right]=$ $\hbar \delta(x-y)$.

It may be observed that for two special cases corresponding to $\eta=0$ and $c=0$, the Hamiltonian of the generalized NLS model (1.1) reduces to that of the NLS model and the derivative NLS (DNLS) model, respectively. The quantum bound states for these integrable NLS and DNLS models have been investigated earlier by using both algebraic Bethe ansatz and coordinate Bethe ansatz [1-4,7-12]. In this Letter, our aim is to investigate the ranges of values of the coupling constants for which localized quantum $N$-body bound states exist for the generalized NLS model. In this context it should be noted that, unlike the cases of the NLS and DNLS models, the integrability of the generalized NLS model (1.1) has not been established so far. So the method of algebraic Bethe ansatz cannot be used at present to study this model.

It may also be mentioned that an analysis of the classical NLS model suggests a fascinating application of the spectral analysis for nonlinear operators, which has been studied earlier [16]. However, for the generalized NLS model, we shall take the following approach. Rather than trying to deal directly with the spectral analysis of the model defined in (1.1), we shall project the second quantized Hamiltonian to the bosonic $N$-particle subspace and reduce it to a linear operator. The method of coordinate Bethe ansatz can then be used to find the spectrum of such a linear operator. In Section 2, we apply this coordinate Bethe ansatz to find out the conditions which the Bethe momenta have to satisfy in order that a quantum $N$-body bound state should exist. In Section 3, we analyze these conditions on Bethe momenta and find that such bound states exist for all possible values (both positive and negative) of $c$ and within several nonoverlapping ranges (called bands) of $\eta$. We also apply the idea of Farey sequences in number theory to completely determine the ranges of all bands for which $N$-body bound states exist for a given value of $N$. In Section 4, we show that the bound states appearing within each band can have both positive and negative momentum. Moreover, bound states with zero momentum can be constructed for a wide range of the coupling constants. We also calculate the binding energy of the bound states and find that it can take both positive and negative value within each band. In Section 5 we make some concluding remarks.

\section{Conditions for quantum $N$-body bound states in the generalized NLS model}

To apply the coordinate Bethe ansatz, we separate the full bosonic Fock space associated with the Hamiltonian (1.1) into disjoint $N$-particle subspaces $\left|S_{N}\right\rangle$. We want to solve the eigenvalue equation $H\left|S_{N}\right\rangle=$ $E\left|S_{N}\right\rangle$. The coordinate representation of this equation is given by

$H_{N} \tau_{N}\left(x_{1}, x_{2}, \ldots, x_{N}\right)=E \tau_{N}\left(x_{1}, x_{2}, \ldots, x_{N}\right)$,

where the $N$-particle symmetric wave function $\tau_{N}\left(x_{1}\right.$, $\left.x_{2}, \ldots, x_{N}\right)$ is defined as

$\tau_{N}\left(x_{1}, x_{2}, \ldots, x_{N}\right)=\frac{1}{\sqrt{N !}}\left\langle 0\left|\psi\left(x_{1}\right) \cdots \psi\left(x_{N}\right)\right| S_{N}\right\rangle$,

and $H_{N}$, the projection of the second-quantized Hamiltonian $H$ (1.1) on to the $N$-particle sector, is given by

$$
\begin{aligned}
H_{N}= & -\hbar^{2} \sum_{j=1}^{N} \frac{\partial^{2}}{\partial x_{j}^{2}}+2 \hbar^{2} c \sum_{l<m} \delta\left(x_{l}-x_{m}\right) \\
& +2 i \hbar^{2} \eta \sum_{l<m} \delta\left(x_{l}-x_{m}\right)\left(\frac{\partial}{\partial x_{l}}+\frac{\partial}{\partial x_{m}}\right) .
\end{aligned}
$$


It is evident that $H_{N}$ commutes with the total momentum operator in the $N$-particle sector, which is defined as

$P_{N}=-i \hbar \sum_{j=1}^{N} \frac{\partial}{\partial x_{j}}$.

Note that $H_{N}$ remains invariant while $P_{N}$ changes sign if we change the sign of $\eta$ and transform all the $x_{i} \rightarrow-x_{i}$, keeping $c$ unchanged; let us call this the parity transformation. Hence it is sufficient to study the model for $\eta>0$. The eigenfunctions for $\eta<0$ can then by obtained by changing $x_{i} \rightarrow-x_{i}$; this leaves the energy invariant but reverses the momentum.

Let us first construct the eigenfunctions of the Hamiltonian (2.3) for the two-particle case, without imposing any symmetry property on $\tau_{2}\left(x_{1}, x_{2}\right)$ under the exchange of the particle coordinates. For the region $x_{1}<x_{2}$, we may take the eigenfunction to be

$\tau_{2}\left(x_{1}, x_{2}\right)=\exp \left\{i\left(k_{1} x_{1}+k_{2} x_{2}\right)\right\}$

where $k_{1}$ and $k_{2}$ are two distinct wave numbers. Using Eq. (2.1) for $N=2$, we find that this two-particle wave function takes the following form in the region $x_{1}>x_{2}$ :

$$
\begin{aligned}
\tau_{2}\left(x_{1}, x_{2}\right)= & A\left(k_{1}, k_{2}\right) \exp \left\{i\left(k_{1} x_{1}+k_{2} x_{2}\right)\right\} \\
& +B\left(k_{1}, k_{2}\right) \exp \left\{i\left(k_{2} x_{1}+k_{1} x_{2}\right)\right\},
\end{aligned}
$$

where the 'matching coefficients' $A\left(k_{1}, k_{2}\right)$ and $B\left(k_{1}, k_{2}\right)$ are given by [7]

$A\left(k_{1}, k_{2}\right)=\frac{k_{1}-k_{2}+i \eta\left(k_{1}+k_{2}\right)-i c}{k_{1}-k_{2}}$,
$B\left(k_{1}, k_{2}\right)=1-A\left(k_{1}, k_{2}\right)$.

By using these matching coefficients, we can construct completely symmetric $N$-particle eigenfunctions for the Hamiltonian (2.3). In the region $x_{1}<x_{2}<\cdots<$ $x_{N}$, these eigenfunctions are given by $[7,17]$

$$
\begin{aligned}
& \tau_{N}\left(x_{1}, x_{2}, \ldots, x_{N}\right) \\
& =\sum_{\omega}\left(\prod_{l<m} \frac{A\left(k_{\omega(m)}, k_{\omega(l)}\right)}{A\left(k_{m}, k_{l}\right)}\right) \\
& \quad \times \rho_{\omega(1), \omega(2), \ldots, \omega(N)}\left(x_{1}, x_{2}, \ldots, x_{N}\right),
\end{aligned}
$$

where

$$
\begin{aligned}
& \rho_{\omega(1), \omega(2), \ldots, \omega(N)}\left(x_{1}, x_{2}, \ldots, x_{N}\right) \\
& \quad=\exp \left\{i\left(k_{\omega(1)} x_{1}+\cdots+k_{\omega(N)} x_{N}\right)\right\} .
\end{aligned}
$$

In the expression (2.8), the $k_{n}$ 's are all distinct wave numbers, and $\sum_{\omega}$ implies summing over all permutations of the integers $(1,2, \ldots, N)$. The eigenvalues of the momentum (2.4) and Hamiltonian (2.3) operators, corresponding to the eigenfunctions $\tau_{N}\left(x_{1}, x_{2}, \ldots\right.$, $x_{N}$ ), are given by

$$
\begin{aligned}
P_{N} & \tau_{N}\left(x_{1}, x_{2}, \ldots, x_{N}\right) \\
= & \hbar\left(\sum_{j=1}^{N} k_{j}\right) \tau_{N}\left(x_{1}, x_{2}, \ldots, x_{N}\right), \\
H_{N} & \tau_{N}\left(x_{1}, x_{2}, \ldots, x_{N}\right) \\
& =\hbar^{2}\left(\sum_{j=1}^{N} k_{j}^{2}\right) \tau_{N}\left(x_{1}, x_{2}, \ldots, x_{N}\right) .
\end{aligned}
$$

The wave function in (2.8) will represent a localized bound state if it decays when any of the relative coordinates measuring the distance between a pair of particles tends towards infinity. To obtain the condition for the existence of such a localized bound state, let us consider the following wave function in the region $x_{1}<x_{2}<\cdots<x_{N}$ :

$\rho_{1,2, \ldots, N}\left(x_{1}, x_{2}, \ldots, x_{N}\right)=\exp \left(i \sum_{j=1}^{N} k_{j} x_{j}\right)$.

As before, the momentum eigenvalue corresponding to this wave function is given by $\hbar \sum_{j=1}^{N} k_{j}$. Since this must be a real quantity, we obtain the condition

$\sum_{j=1}^{N} q_{j}=0$,

where $q_{j}$ denotes the imaginary part of $k_{j}$. The probability density corresponding to the wave function $\rho_{1,2, \ldots, N}\left(x_{1}, x_{2}, \ldots, x_{N}\right)$ in (2.11) can be expressed as

$$
\begin{aligned}
& \left|\rho_{1,2, \ldots, N}\left(x_{1}, x_{2}, \ldots, x_{N}\right)\right|^{2} \\
& \quad=\exp \left\{2 \sum_{r=1}^{N-1}\left(\sum_{j=1}^{r} q_{j}\right) y_{r}\right\},
\end{aligned}
$$

where the $y_{r}$ 's are the $N-1$ relative coordinates: $y_{r} \equiv x_{r+1}-x_{r}$, and we have used Eq. (2.12). It is evident that the probability density in (2.13) decays exponentially in the limit $y_{r} \rightarrow \infty$ for one or more values of $r$, provided that all the following conditions 
are satisfied:

$q_{1}<0, \quad q_{1}+q_{2}<0, \quad \ldots, \quad \sum_{j=1}^{N-1} q_{j}<0$.

Eqs. (2.12) and (2.14) imply that the wave function $\rho_{1,2, \ldots, N}\left(x_{1}, x_{2}, \ldots, x_{N}\right)(2.11)$ is square-integrable if one holds the centre-of-mass coordinate $X=\sum_{i} x_{i} / N$ fixed, and integrates over the relative coordinates $y_{r}$. In the region $x_{1}<x_{2}<\cdots<x_{N}$, the integrals over the $y_{r}$ 's all run from 0 to $\infty$, and they are independent of each other. The probability density (2.13) is independent of $X$ and due to the conditions (2.14), the integration of this probability density over the $y_{r}$ 's gives a finite result.

Note that the wave function (2.11) is obtained by taking $\omega$ as the identity permutation in (2.9). However, the full wave function (2.8) also contains terms like (2.9) with $\omega$ representing all possible nontrivial permutations. The conditions which ensure the decay of such a term with a nontrivial permutation will, in general, contradict the conditions (2.14). To construct a decaying wave function, therefore, the coefficients of all terms $\rho_{\omega(1), \omega(2), \ldots, \omega(N)}\left(x_{1}, x_{2}, \ldots, x_{N}\right)$ with nontrivial permutations must be made to vanish. It turns out that it is sufficient to require:

$$
\begin{gathered}
A\left(k_{1}, k_{2}\right)=0, \quad A\left(k_{2}, k_{3}\right)=0, \\
\ldots, \quad A\left(k_{N-1}, k_{N}\right)=0 .
\end{gathered}
$$

Thus the simultaneous validity of the conditions (2.12), (2.14) and (2.15) ensures that the full wave function $\tau_{N}\left(x_{1}, x_{2}, \ldots, x_{N}\right)$ (2.8) represents a localized bound state. Using the conditions (2.12) and (2.15), one can obtain an expression for the complex $k_{n}$ 's in the form

$k_{n}=\chi e^{-i(N+1-2 n) \phi}+\frac{c}{2 \tan \phi}$,

where $\chi$ is a real parameter, and $\phi$ is related to the coupling constant as

$\phi=\tan ^{-1}(\eta)$.

To obtain an unique value of $\phi$ from the above equation, we restrict it to the fundamental region $-\frac{\pi}{2}<$ $\phi(\neq 0)<\frac{\pi}{2}$. [Note that $\eta$ and $\phi$ have the same sign. Due to the parity symmetry mentioned above, we can restrict our attention to the range $0<\phi<\frac{\pi}{2}$.]
Now, let us verify whether the $k_{n}$ 's in (2.16) satisfy the conditions (2.14). Summing over the imaginary parts of these $k_{n}$ 's, we can express the conditions (2.14) in the form

$$
\begin{gathered}
\chi \frac{\sin (l \phi)}{\sin \phi} \sin [(N-l) \phi]>0 \\
\text { for } l=1,2, \ldots, N-1 .
\end{gathered}
$$

Thus, for some given values of $c, \phi, N$ and $\chi$, a bound state will exist when all the above inequalities are simultaneously satisfied. Surprisingly, the above condition for having a quantum bound state for the generalized NLS model is completely independent of the coupling constant $c$. By using Eqs. (2.16) and (2.10a), we obtain the momentum eigenvalue of such a bound state to be

$P=\frac{\hbar c N}{2 \tan \phi}+\hbar \chi \frac{\sin (N \phi)}{\sin \phi}$.

Hence $\chi$ is related to the momentum as

$\chi=\frac{1}{\hbar}\left(P-\frac{\hbar c N}{2 \tan \phi}\right) \frac{\sin \phi}{\sin (N \phi)}$.

In a similar way, the energy eigenvalue of these bound states is found to be

$$
\begin{aligned}
E= & \frac{\hbar^{2} \chi^{2} \sin (2 N \phi)}{\sin (2 \phi)}+\frac{\hbar^{2} c^{2} N}{4 \tan ^{2} \phi} \\
& +\frac{\hbar^{2} \chi c \sin (N \phi) \cos \phi}{\sin ^{2} \phi} .
\end{aligned}
$$

For some fixed value of $c, \phi$ and $N$, the above expression for the energy has a minimum for a nonzero value of the momentum given by

$P_{0}=\frac{N \hbar c}{2 \tan \phi}\left(1-\frac{\tan (N \phi)}{N \tan \phi}\right)$,

provided that $\tan (N \phi)>0$. The next section of our Letter will be devoted to finding the ranges of values of $\phi$ where all the inequalities (2.18) are simultaneously satisfied for a given value of the particle number $N$.

\section{Determining the values of $\phi$ where $N$-body bound states exist}

In this section, we will study the values of $\phi$ where $N$-body bound states exist for different values of $N$ 
for the generalized NLS model. In an earlier analysis, the quantum bound states of this model had been found to exist for $0<\phi<\frac{\pi}{N}$ [7]. However, from our analysis, it will turn out that there are several nonoverlapping ranges (bands) of $\phi$ for which quantum bound states exist for the generalized NLS model. Even the lowest band for which such bound states exist (i.e., when $\phi$ lies in the range $0<\phi<\frac{\pi}{N-1}$ ) is wider than that obtained earlier [7]. Since Eq. (2.18) is independent of the coupling constant $c$, bound states are formed within the above mentioned bands for all possible values of $c$. Consequently, in contrast to the case of NLS model where bound states appear only for negative values of $c$, bound states of the generalized NLS model can appear for both positive and negative values of $c$.

Incidentally, it is quite remarkable that our results concerning the band structure of the generalized NLS model turn out to be very similar to our analysis on the quantum bound states of the DNLS model $[11,12]$. For the simplest case $N=2$, the condition (2.18) is satisfied when $\phi$ lies in the range $0<\phi<\frac{\pi}{2}\left(-\frac{\pi}{2}<\right.$ $\phi<0)$ for the choice $\chi>0(\chi<0)$. Thus any nonzero value of $\phi$ within its fundamental region can generate a 2-body bound state.

We will now consider the more interesting case with $N \geqslant 3$. Due to the parity symmetry of the Hamiltonian in (2.3), we will henceforth assume that $\phi>0$.

Let us consider a value of $\phi$ of the form

$\phi_{N, n} \equiv \frac{\pi n}{N}$,

where $n$ is an integer satisfying $1 \leqslant n<N / 2$.

It can be easily shown that all the inequalities in (2.18) are satisfied for $\phi=\phi_{N, n}$, if and only if $N$ and $n$ are relatively prime (with $n$ odd for $\chi>0$, and $n$ even for $\chi<0$ ) [12]. By continuity, it then follows that all the inequalities will hold in a neighborhood of $\phi_{N, n}$ extending from a value $\phi_{N, n,-}$ to a value $\phi_{N, n,+}$, such that $\phi_{N, n,-}<\phi_{N, n}<\phi_{N, n,+}$. We will call the region

$\phi_{N, n,-}<\phi<\phi_{N, n,+}$

as the band $B_{N, n}$. In this band, there is a bound state with $N$ particles.

We now have to determine the end points $\phi_{N, n,-}$ and $\phi_{N, n,+}$ of the band $B_{N, n}$. The inequalities in (2.18) show that the end points are given by $\phi$ of the form

$\phi=\frac{\pi j}{l}$,

where $j$ and $l$ are relatively prime and satisfy the conditions:

$1 \leqslant l<N \quad$ and $\quad j<\frac{l}{2}$.

Thus the end points of the band $B_{N, n}$ are given by two rational numbers $\phi / \pi$ of the form $j / l$ which lie closest to (and on either side of) the point $\phi_{N, n} / \pi=$ $n / N$. The solution to this problem is well known in number theory and is described by the Farey sequences [18].

For a positive integer $N$, the Farey sequence $F_{N}$ is defined to be the set of all the fractions $a / b$ in increasing order such that (i) $0 \leqslant a \leqslant b \leqslant N$, and (ii) $a$ and $b$ are relatively prime.

The Farey sequences for the first few integers are given by

$$
\begin{array}{llllllllllllll}
F_{1}: & \frac{0}{1} & \frac{1}{1} & & & & & & & & \\
F_{2}: & \frac{0}{1} & \frac{1}{2} & \frac{1}{1} & & & & & & & \\
F_{3}: & \frac{0}{1} & \frac{1}{3} & \frac{1}{2} & \frac{2}{3} & \frac{1}{1} & & & & & \\
F_{4}: & \frac{0}{1} & \frac{1}{4} & \frac{1}{3} & \frac{1}{2} & \frac{2}{3} & \frac{3}{4} & \frac{1}{1} & & & & \\
F_{5}: & \frac{0}{1} & \frac{1}{5} & \frac{1}{4} & \frac{1}{3} & \frac{2}{5} & \frac{1}{2} & \frac{3}{5} & \frac{2}{3} & \frac{3}{4} & \frac{4}{5} & \frac{1}{1}
\end{array}
$$

To return to our problem, we now see that the points $\phi_{N, n}$ in (3.1) (which lie in the bands $B_{N, n}$ ) have a oneto-one correspondence with the fractions $n / N$, which appear on the left side of $1 / 2$ within the sequence $F_{N}$. Due to Eqs. (3.3) and (3.4), the end points of the band $B_{N, n}$ are given by

$\phi_{N, n,-}=\frac{\pi a_{1}}{b_{1}}, \quad \phi_{N, n,+}=\frac{\pi a_{2}}{b_{2}}$,

where $a_{1} / b_{1}$ and $a_{2} / b_{2}$ are the fractions lying immediately to the left and right of $n / N$ in the Farey sequence $F_{N}$. These are the two unique fractions which lie closest to (and on either side) of $n / N$.

In Table 1, we show the ranges of values of $\phi$ for which bound states exist for $N=2$ to 9 . 
Table 1

The range of values of $\phi / \pi$ for which bound states exist for various values of $N$

\begin{tabular}{lcc}
\hline$N$ & $n$ & Range of values of $\phi / \pi$ \\
\hline 2 & 1 & $0<\phi / \pi<1 / 2$ \\
3 & 1 & $0<\phi / \pi<1 / 2$ \\
4 & 1 & $0<\phi / \pi<1 / 3$ \\
5 & 1 & $0<\phi / \pi<1 / 4$ \\
5 & 2 & $1 / 3<\phi / \pi<1 / 2$ \\
6 & 1 & $0<\phi / \pi<1 / 5$ \\
7 & 1 & $0<\phi / \pi<1 / 6$ \\
7 & 2 & $1 / 4<\phi / \pi<1 / 3$ \\
7 & 3 & $2 / 5<\phi / \pi<1 / 2$ \\
8 & 1 & $0<\phi / \pi<1 / 7$ \\
8 & 3 & $1 / 3<\phi / \pi<2 / 5$ \\
9 & 1 & $0<\phi / \pi<1 / 8$ \\
9 & 2 & $1 / 5<\phi / \pi<1 / 4$ \\
9 & 4 & $3 / 7<\phi / \pi<1 / 2$ \\
\hline
\end{tabular}

\section{Momentum and binding energy of a $N$-body bound state}

In the previous section, we have determined the band structure associated with the generalized NLS model by proceeding in the same way as in the case of the DNLS model. However it should be noted that, in the case of the DNLS model, one does not get quantum bound states with zero momentum expect for some discrete values of the coupling constant $\phi=\pi n / N$ [12]. Thus, in general, bound states of the DNLS model do not exist in the centre-of-mass (COM) frame. This may be due to the fact that in the Hamiltonian of the DNLS model there is no dimensionful coupling constant, and it is invariant under a scale transformation. On the other hand, the Hamiltonian of the generalized NLS model (1.1) has a dimensionful coupling which breaks the scale invariance. So, we may expect to get zero momentum bound states for this model within a wide range of the coupling constants. The binding energy of such zero momentum states may then be interpreted as their internal energy in the COM frame. With this aim in mind, in this section we will calculate the momentum and binding energy for the $N$-body bound states described above.

We first look at the momentum of the bound states in a particular band $B_{N, n}$ using Eq. (2.19). The form of the end points given in (3.6) shows that $\sin (N \phi)=0$ at only one point in the band $B_{N, n}$, namely, at $\phi=$

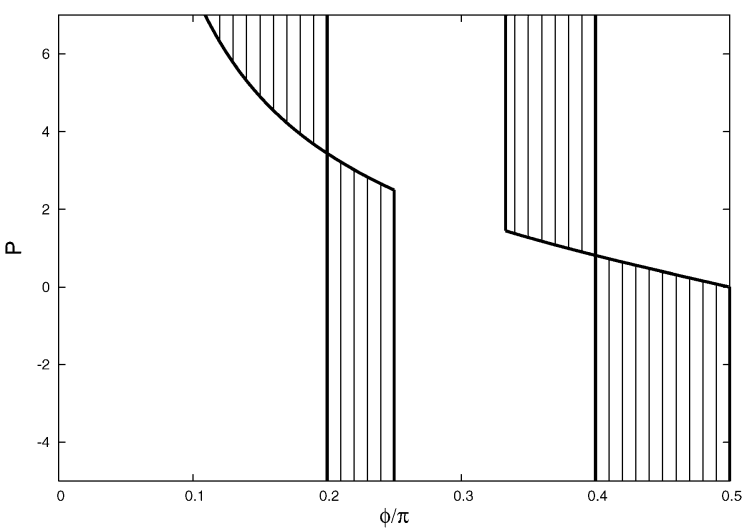

(a)

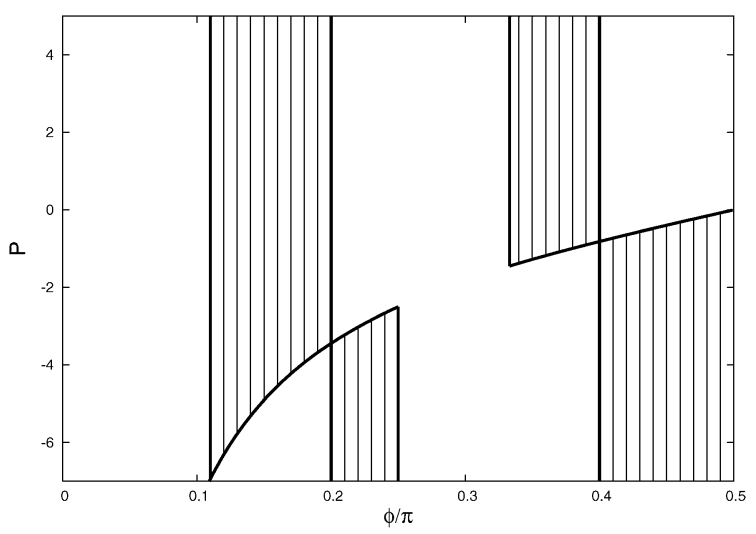

(b)

Fig. 1. The filled in regions indicate the values of the momentum as a function of $\phi / \pi$ for which a 5-body bound state exists. In figures (a) and (b), $\hbar c=1$ and -1 , respectively.

$\phi_{N, n}$. In the right part of the band (i.e., from $\phi_{N, n}$ to $\left.\phi_{N, n,+}\right)$, the sign of $\sin (N \phi)$ is $(-1)^{n}$. In the left part of the band (i.e., from $\phi_{N, n,-}$ to $\phi_{N, n}$ ), the sign of $\sin (N \phi)$ is $(-1)^{n+1}$. Now, the analysis given above showed that $\chi$ has the same sign as $(-1)^{n+1}$. Hence the momentum given in (2.19) is greater (less) than $\hbar c N /(2 \tan \phi)$ in the left (right) part of a band, and is equal to $\hbar c N /(2 \tan \phi)$ at $\phi=\phi_{N, n}$.

In Figs. 1a and b, we have plotted the allowed values of momentum $(P)$ (2.19) as a function of $\phi / \pi$ for $N=5$. In Fig. 1a (taking $\hbar c=1)$ the minimum (maximum) value of $P$ in the left (right) part of a band is $5 / 2 \tan \phi$. Similarly, in Fig. $1 \mathrm{~b}$ (taking $\hbar c=-1$ ) the minimum (maximum) value of $P$ in the left (right) part of a band is $-5 / 2 \tan \phi$. 
Next, we look at the energy using Eq. (2.21). To calculate the binding energy, we consider a reference state in which the momentum $P$ of the $N$-body bound state given in (2.19) is equally distributed amongst $N$ single-particle scattering states. The real wave number associated with each of these single-particle states is denoted by $k_{0}$. From Eqs. (2.10a) and (2.19), we obtain

$k_{0}=\frac{c}{2 \tan \phi}+\frac{\chi \sin (N \phi)}{N \sin \phi}$.

Using Eq. (2.10b), we can calculate the total energy for the $N$ single-particle scattering state as

$$
\begin{aligned}
E_{S}= & \hbar^{2} N k_{0}^{2}=\frac{\hbar^{2} \chi^{2} \sin ^{2}(N \phi)}{N \sin ^{2} \phi}+\frac{\hbar^{2} c^{2} N}{4 \tan ^{2} \phi} \\
& +\frac{\hbar^{2} \chi c \sin (N \phi) \cos \phi}{\sin ^{2} \phi} .
\end{aligned}
$$

Subtracting $E$ in (2.21) from $E_{S}$ in (4.2), we obtain the binding energy of the $N$-body bound state as

$$
\begin{aligned}
E_{B}(\phi, N) & \equiv E_{s}-E \\
& =\frac{\hbar^{2} \chi^{2} \sin (N \phi)}{\sin \phi}\left\{\frac{\sin (N \phi)}{N \sin \phi}-\frac{\cos (N \phi)}{\cos \phi}\right\} .
\end{aligned}
$$

It may be noted that the above expression for the binding energy remains invariant under the transformation $\phi \rightarrow-\phi$. Now, it can be easily shown that this expression for the binding energy is positive in the left part of each band, negative in the right part, and zero at the point $\phi=\phi_{N, n}$ [12].

We thus see that for $\phi>0,(P-\hbar c N / 2 \tan \phi)$ and the binding energy are both positive in the left part of a band, and they are both negative in the right part of a band. Consequently, for the case $\phi>0$ and $c>0$ $(c<0)$, bound states with zero momentum can be constructed within the right (left) side of each band. Using Eq. (2.18) it is easy to see that, the momentum of a quantum bound state vanishes for

$$
\chi=-\frac{c N}{2} \frac{\cos \phi}{\sin (N \phi)} \text {. }
$$

Substituting this value of $\chi$ to Eq. (4.3), we obtain the binding energy for these zero momentum bound states as

$$
E_{B}^{P=0}(\phi, N)=\frac{\hbar^{2} c^{2} N}{4 \tan ^{2} \phi}\left[1-\frac{N \tan \phi}{\tan (N \phi)}\right] .
$$

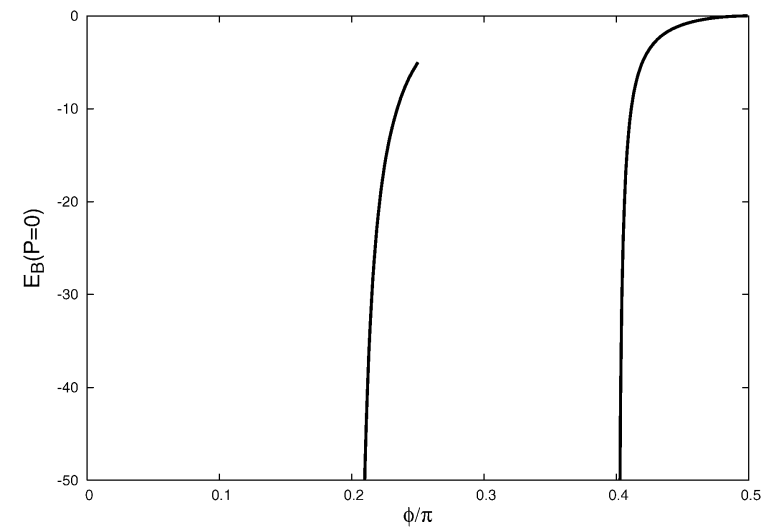

(a)

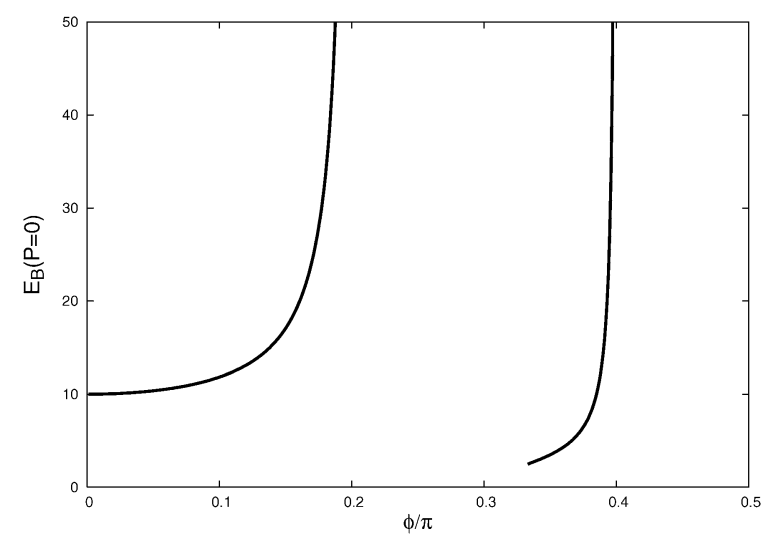

(b)

Fig. 2. The binding energy of the zero momentum 5-body bound state as a function of $\phi / \pi$. In figures (a) and (b), $\hbar c=1$ and -1 , respectively.

This can be interpreted as the internal energy of a quantum bound state in its COM frame.

In Figs. 2a and b, we have plotted the above expression for the binding energy (4.5) as a function of $\phi / \pi$ for $N=5$ ( $\hbar c=1$ in Fig. $2 \mathrm{a}$ and $\hbar c=-1$ in Fig. 2b).

Finally, we want to discuss a subtle connection between quantum bound states of the generalized NLS model and the ordinary NLS model. It has been already mentioned that, in the limit $\phi \rightarrow 0$, the ordinary NLS model can be obtained from the generalized NLS model. It is well known that quantum bound states of NLS model exist only for $c<0$ with the $N$ momenta being given by

$k_{n}=\frac{P}{N \hbar}+i \frac{c}{2}(N+1-2 n)$, 
and the binding energy for such state taking the form

$E_{B}(N)=\hbar^{2} c^{2} N\left(N^{2}-1\right) / 12$.

On the other hand, in this article we have seen that, for any small value of the parameter $\phi$ lying within the lowest band, quantum bound states of the generalized NLS model exist for both $c>0$ and $c<0$. So it is natural to ask why bound states corresponding to $c>0$ disappear in the limit $\phi \rightarrow 0$. To answer this question, we first try to take the $\phi \rightarrow 0$ limit of the complex momenta $k_{n}(2.16)$ associated with the generalized NLS model and check whether they reduce to the $k_{n}$ given in Eq. (4.6). However, it is evident that, all $k_{n}$ in Eq. (2.16) as well as the corresponding total momentum $P$ (2.19) will diverge if we take the limit $\phi \rightarrow 0$ by keeping $\chi$ fixed. To bypass this problem, we have to take the limit $\phi \rightarrow 0$ in a way such that the total momentum $P(2.19)$ remains constant. For the sake of convenience we choose $P=0$, for which the value of $\chi$ is given in Eq. (4.4). Substituting this value of $\chi$ in Eq. (2.16) and expanding the right-hand side of this equation in a power series of $\phi$, we easily obtain

$k_{n}=i \frac{c}{2}(N+1-2 n)+\mathcal{O}(\phi)$,

which reproduces the value of $k_{n}$ in Eq. (4.6) for $P=0$. Similarly, by taking the limit $\phi \rightarrow 0$ of the binding energy (4.5) corresponding to the zero momentum bound states of the generalized NLS model, we can reproduce $E_{B}(N)$ in Eq. (4.7). In the case of the generalized NLS model with $c>0$, we have already observed that zero momentum bound states exist only in the right side of the each band. For taking the limit $\phi \rightarrow 0$, however, we have to move to the left side of the lowest band. Consequently, it is impossible to take the limit $\phi \rightarrow 0$ within the lowest band after fixing $P=0$ (same conclusion can be drawn for any other fixed value of $P$ ). On the other hand, for $c<0$, zero momentum bound states exist in the left side of each band and one can easily take the limit $\phi \rightarrow 0$ after fixing $P=0$. This fact clearly explains why quantum bound states remain in the limit $\phi \rightarrow 0$ only if $c<0$.

\section{Conclusion}

By applying the coordinate Bethe ansatz, we have investigated the range of the coupling constants $(c, \phi)$ for which localized quantum $N$-body bound states exist in a generalized NLS model. It is found that such bound states exist for all possible values (both positive and negative) of $c$ and within several nonoverlapping ranges (called bands) of $\phi$. Using the ideas of Farey sequences appearing in number theory, we have given explicit expressions for all the allowed bands of $\phi$ for which $N$-body bound states exist.

We have also calculated the momentum and binding energy for the bound states within all bands in the region $\phi>0$. It is found that bound states with positive (negative) values of $(P-\hbar c N / 2 \tan \phi)$ appear in the left (right) part of each band, and these states have positive (negative) binding energy. Consequently, bound states with zero momentum can be constructed within a wide range of the coupling constants. This happens in contrast to the case of the DNLS model, where zero momentum bound states appear for only some discrete values of $\phi$. The binding energy of zero momentum states has also been calculated and interpreted as the internal energy in the centre-of-mass frame. We have also remarked on some subtleties of taking the limit $\phi \rightarrow 0$ for the bound states of the generalized NLS model, and explained why this process does not lead to any bound state of NLS model for $c>0$.

It is currently not known if the generalized NLS model discussed here is integrable. If the model turns out to be nonintegrable, then the bound states which we have found using the Bethe ansatz may not be complete, and there could be some other bound states. This may be an interesting problem for future studies.

\section{References}

[1] J.B. McGuire, J. Math. Phys. 5 (1964) 622;

F.A. Berezin, G.P. Pochil, V.M. Finkelberg, Moscow Univ. Vestnik 1 (1964) 21;

F. Calogero, A. Degasperis, Phys. Rev. A 11 (1975) 265.

[2] H.B. Thacker, Rev. Mod. Phys. 53 (1981) 253.

[3] L.D. Faddeev, Sov. Sci. Rev. C 1 (1980) 107;

L.D. Faddeev, in: J.-B. Zuber, R. Stora (Eds.), Recent Advances in Field Theory and Statistical Mechanics, NorthHolland, Amsterdam, 1984, p. 561.

[4] E.K. Sklyanin, in: M. Jimbo (Ed.), Yang-Baxter Equation in Integrable Systems, in: Advanced Series in Mathematical Physics, vol. 10, World Scientific, Singapore, 1990, p. 121.

[5] E.K. Sklyanin, L.A. Takhtajan, L.D. Faddeev, Theor. Math. Phys. 40 (1980) 688.

[6] G. Bhattacharya, S. Ghosh, Int. J. Mod. Phys. A 4 (1989) 627. 
[7] A.G. Shnirman, B.A. Malomed, E. Ben-Jacob, Phys. Rev. A 50 (1994) 3453.

[8] A. Kundu, B. Basu-Mallick, J. Math. Phys. 34 (1993) 1052.

[9] B. Basu-Mallick, T. Bhattacharyya, Nucl. Phys. B 634 (2002) 611.

[10] B. Basu-Mallick, T. Bhattacharyya, Nucl. Phys. B 668 (2003) 415.

[11] B. Basu-Mallick, T. Bhattacharyya, D. Sen, Phys. Lett. A 325 (2004) 375 .

[12] B. Basu-Mallick, T. Bhattacharyya, D. Sen, Nucl. Phys. B 675 (2003) 516.

[13] M. Wadati, H. Sanuki, K. Konno, Y.-H. Ichikawa, Rocky Mountain J. Math. 8 (1978) 323;

Y.-H. Ichikawa, S. Watanabe, J. Physique C 38 (1977) 6;
Y.-H. Ichikawa, K. Konno, M. Wadati, H. Sanuki, J. Phys. Soc. Jpn. 48 (1980) 279.

[14] P.A. Clarkson, Nonlinearity 5 (1992) 453.

[15] Y. Kodama, A. Hasegawa, IEEE J. Quantum Electron. QE-23 (1987) 510.

[16] P.H. Rabinowitz, Commun. Pure Appl. Math. 23 (1970) 939; P.H. Rabinowitz, J. Funct. Anal. 7 (1971) 487;

J. Appel, M.D. Dorfner, Nonlinear Anal. Theor. Methods Appl. 28 (1997) 1955;

J. Appel, Nonlinear Anal. Theor. Methods Appl. 30 (1997) 3135.

[17] E. Gutkin, Ann. Phys. 176 (1987) 22.

[18] I. Niven, H.S. Zuckerman, H.L. Montgomery, An Introduction to the Theory of Numbers, Wiley, New York, 2000. 